\title{
Valoración Médico Deportiva: Aspectos Biopsicosociales relacionados con las Actividades Físicas y Deportivas en Niños y Adolescentes
}

\author{
Ramiro E. Guzmán Guzmánª
}

\author{
a Médico Especialista en \\ Medicina Familiar. Diplomado \\ en Educación Superior. \\ Diplomado en Psicopedago- \\ gía, Planificación, Evaluación \\ y Gestión de la Educación \\ Superior en Salud \\ (P.P.E.G.E.S.S).
}

Correspondencia: Ramiro Guzmán Guzmán, Caja Nacional de Salud, distrital La Paz-Bolivia.

Correo electrónico: guzman_ramiro@hotmail.com

Recibido el 24 de julio de 2010.

Aceptado para su publicación el 7 de septiembre de 2010.

\begin{abstract}
RESUMEN
El desarrollo de un estilo de vida físicamente activo constituye un objetivo para todos los niños y adolescentes. Se ha demostrado que la actividad física deportiva reporta beneficios indudables para la salud y hay estudios de corte transversal que asocian la actividad deportiva con el menor consumo de tabaco y alcohol, así como el aumento de la masa mineral ósea y pérdida de peso en niños y adolescentes con obesidad.

Una de las tareas del médico de atención primaria es tomar parte en los programas de salud para la infancia y la adolescencia realizando revisiones específicas y recomendaciones sobre la actividad física, estilos de vida y prevención de lesiones. También se recomienda una revisión especifica para niños y adolescentes que realicen deportes, por considerarse una fuente de salud y desarrollo para el hombre y un espíritu de iniciativa y responsabilidad que le permita expresarse y superarse a la vez que los disciplina, aumenta su rendimiento y los integra al mundo social.

Palabras clave. Medicina Deportiva, Ejercicio.
\end{abstract}

\section{ABSTRACT}

Sports Medical Assessment: Biopsychosocial Aspects related to Sports and Physical Activity in Children and Adolescents

The development of a physically active lifestyle is an objective for all children and adolescents. It has been demonstrated that physical activity is undoubtedly beneficial to health and there are cross-sectional studies that associate the practice of a sport with a lower consumption of cigarettes and alcohol, as well as increased bone mass and weight loss in obese children and adolescents.

One of the tasks of the primary care physician is to take part in health programs for children and adolescents by providing recommendations for physical activity, lifestyle and injury prevention. It is also recommended that children and adolescents practice sports as this is, seen as a source of health and human development and encourages a spirit of initiative and responsibility that will enable children to express themselves improve themselves. At the same time it will discipline them, increase their performance and help them to become socially integrated.

Key words. Sports Medicine, Exercise.

\section{INTRODUCCIÓN}

La práctica de la actividad física es definida como cualquier movimiento corporal voluntario de contracción muscular, con gasto energético mayor al de reposo, y entendida como un comportamiento humano complejo, voluntario y autónomo, con componentes y determinantes de orden biológico y psicosocio-cultural. Ejemplificada por deportes, ejercicios físicos, bailes y determinadas actividades de recreación y cotidianas, es un derecho fundamental que todos los seres humanos tienen para su completo desarrollo físico, mental y social. 
El desarrollo físico, social y mental de los individuos, la promoción de la salud y prevención de las enfermedades y el mejoramiento o mantenimiento de la calidad de vida social y laboral dependen no sólo de las entidades gubernamentales sino de todos los integrantes de la sociedad. La actividad física, el ejercicio y el deporte son elementos preventivos y en muchas ocasiones terapéuticos que permitirán coadyuvar en unión con otros hábitos de vida sana para una mejor sociedad, más sana, fuerte y saludable.

\section{ASPECTOS GENERALES}

1. Todos los niños en edad preescolar y escolar deberían participar regularmente en algún tipo de actividad física adecuada a su grado de desarrollo y salud física, llegando a largo plazo a adoptar dichas actividades deportivas como parte de su estilo de vida.

2. En general no se ha determinado el tipo y la cantidad de ejercicio para conseguir una óptima capacidad funcional y de salud en los niños escolares:

- En los niños preescolares la actividad innata hace que probablemente alcancen unos niveles adecuados de aptitud física cuado se les permite expresar su curiosidad y exploración espontáneas. Bajo estas circunstancias suelen ser innecesarias intervenciones específicas para mejorar su aptitud física.

- En escolares y adolescentes se recomienda al menos 20 minutos de actividad física, al menos tres veces por semana.

3. El médico debe alentar hacia esta participación e incorporar una evaluación de la actividad física en la historia clínica. Las citas de los controles de salud deben incluir:

- Intereses y participación en actividades físicas organizadas o no.

- Tiempo dedicado a ver televisión en la semana.

- Hábitos de ejercicios de otros miembros de la familia (debe alentarse a la participación de los padres en programas de actividad física y proporcionar asesoria a la familia y la escuela).

4. Debe alentarse la actividad física como actividad natural que forme parte de un estilo de vida saludable y no como meta para aumentar el desarrollo motor o la capacidad deportiva del niño.

5. El juego libre diseñado en edad preescolar y escolar temprana es preferible a sesiones estructurales.

6. La capacitación para participar en deportes organizados debe determinarse de forma individual y basándose en el deseo del niño (no de los padres), siendo aconsejable siempre después de los 6 años.

7. En los programas deportivos estructurados debe primar el objetivo de la participación y el disfrute frente a los de competición y victoria. El deporte organizado asegura la actividad física regular.

8. Debe alentarse a padres y otros miembros del grupo familiar a que sirvan como modelo para la participación de sus hijos en programas de actividad física, así como alentarlos a realizar con sus hijos actividades físicas comunes.

9. La recomendación de la GAPS (Guidelines of Adolescent Preventive Services) es la realización de visitas preventivas anualmente y dentro de ellas el abordaje sistemático de la actividad física, estilos de vida saludables y prevención de lesiones.

10. Las recomendaciones, indicaciones y limitaciones acerca de la participación deportiva en diversas patologías están consensuadas según las diversas enfermedades y según el tipo de deporte clasificado según sea más o menos dinámico.

\section{PARTICIPACIÓN EN DEPORTES ORGANIZA- DOS}

En general debemos tener en cuenta que el beneficio deportivo se obtiene siempre de la expectación y la demanda no excederá la maduración y preparación del niño. Para poder optimizar la seguridad y los beneficios, debemos seguir las siguientes recomendaciones:

- El deporte organizado debe complementar y no reemplazar la actividad física regular que debiera siempre ser aconsejada.

- El médico debe aconsejar sobre el grado de desarrollo físico y aspectos derivados del examen médico, de las condiciones sociales y de maduración cognitiva, para ver si son adecuadas 
en la participación de actividades deportivas específicas.

- Deberá el médico de atención primaria intervenir aconsejando a entrenadores u otro personal relacionado sobre el desarrollo y seguridad del deporte organizado, participando en comités y asociaciones deportivas, así como en programas de seguridad en el deporte, y evaluar cuándo la presión del deporte organizado es excesiva.

- También deberá recomendar el momento óptimo para comenzar a participar en deportes organizados, identificar los riesgos y establecer estrategias de entrenamiento seguras, educar además al personal encargado (entrenadores, profesores de educación física, etc.) sobre las características del niño y adolescente, además de desarrollar en forma conjunta estrategias de prevención de lesiones.

\section{ASPECTOS RELACIONADOS CON LOS BENE- FICIOS Y RIESGOS ASOCIADOS AL DEPORTE INFANTO - JUVENIL ORGANIZADO}

Los beneficios de la actividad física a lo largo de la vida son indiscutibles, tanto en el plano físico como en el psicológico, social y moral.

Hay quienes afirman que la participación de los niños y los adolescentes en deportes organizados no es, en sí misma, ni buena ni mala. La experiencia positiva o negativa no estará dada por la participación en sí misma, sino por la manera en que los adultos imparten esa experiencia y el juego de muchos factores mediadores como el individual, familiar, los entrenadores y la sociedad.

Por lo tanto, los aspectos de la práctica deportiva por parte de los niños y adolescentes que debemos considerar son de orden biológico, psicológico y social.

\section{Aspectos biológicos.}

Los niños no son adultos en miniatura, son únicos en sus fases de desarrollo. Cuando crecen y aumentan de tamaño maduran también sus capacidades funcionales y así van adquiriendo paulatinamente el desarrollo de su capacidad motora, la fuerza, la capacidad aeróbica y anaeróbica, etc. El estado de madurez de un niño o adolescente puede establecerse por la edad cronológica, la edad ósea y la maduración biológica o madurez sexual.

Huesos. Forman el esqueleto y son los que soportan en gran medida la carga corporal, así como son los responsables del $97 \%$ o $98 \%$ de la talla corporal, del $15 \%$ del peso en el recién nacido y del $16 \%$ o $17 \%$ del peso corporal en el adulto, es decir, que son un importante componente en la estatura, el peso y la composición corporal. Desde el nacimiento el hueso se forma por medio de los procesos de osificación, a partir de un centro de osificación primario ubicado en la parte interna y media de la diáfisis ósea y otros secundarios ubicados en las epífisis. Mientras ocurre la formación del hueso en su parte central, van proliferando rápidamente en ambos extremos de la diáfisis y dejando entre ésta y las epífisis los cartílagos de conjunción o de crecimiento, que completarán el crecimiento en largo del hueso. Si por alguna circunstancia estos cartílagos de crecimiento sufren una lesión, como una fractura o epifisitis traumática, se puede llegar a afectar el proceso normal de crecimiento. Estas lesiones se presentan con mayor facilidad en unos deportes que en otros, como el béisbol, tenis (codo del tenista), natación (hombro del nadador), etc. El ejercicio no afecta prácticamente la longitud de los huesos, pero sí ejerce su acción sobre su ancho y densidad. La edad ósea es una medida muy importante en el desarrollo fisiológico. A diferencia de la edad cronológica, la edad ósea se corresponde muy estrechamente con la maduración general, el desarrollo sexual y la edad de la menarca. La edad ósea se determina mediante radiografía de la mano y de la muñeca. Para determinar la edad ósea se compara una radiografía de la mano y la muñeca izquierda con los estándares de Greulich-Pyle o con el método de Tanner Whitehouse.

Músculos. Los músculos conforman la mayor masa tisular del cuerpo. Son voluntarios o esqueléticos, involuntarios o lisos y el músculo cardíaco. Nos referiremos especialmente a los músculos esqueléticos o voluntarios. Existen más de 500 en todo el cuerpo, compuestos por pequeñas unidades, las fibras musculares. Éstas se clasifican en fibras de contracción lenta y de contracción rápida, con un $50 \%$ de cada tipo aproximadamente; la proporción es diferente en algunos músculos, según la mayor o menor actividad que deban realizar. En general, las fibras de contracción lenta actúan en un medio aeróbico, mientras que las de contracción rápida están mejor adaptadas para rendir anaeróbicamente. La masa muscular aumenta de manera sostenida desde el nacimiento hasta la adolescencia, cons- 
tituyendo en el varón, al llegar a la edad adulta, el $40 \%$ del peso corporal. El momento de mayor desarrollo de la masa muscular ocurre durante la pubertad (grados IV y $\vee$ de Tanner en el varón) debido al aumento de la testosterona. No ocurre lo mismo en el sexo femenino, si bien la masa muscular continúa aumentando en ellas, pero de manera más lenta. El máximo nivel se manifiesta en las niñas entre los 16 y 20 años y en los varones entre los 18 y los 25 años de edad. De acuerdo con el desarrollo de la masa muscular por áreas corporales, la distribución se presenta de la siguiente manera: el peso de la masa muscular en cabeza y tronco representa el $40 \%$ al nacimiento y disminuye al $25 \%$ a $30 \%$ en la madurez. En los miembros inferiores constituye aproximadamente el $40 \%$ del peso total de la musculatura al nacimiento, alcanzando un $55 \%$ en la madurez. Por otro lado, con respecto a los miembros superiores, la proporción se mantiene bastante constante desde el nacimiento hasta la madurez, con un $20 \%$ del total del peso de la masa muscular.

Grasa. El tejido adiposo es el componente estructural y funcional de la masa grasa, la que presenta grandes cambios durante el crecimiento, aumentando gradualmente desde la niñez hasta la adolescencia tanto en el varón como en la niña, con un incremento mayor después de los 8 años de edad. Después de la adolescencia, el promedio de grasa es dos veces mayor en las niñas que en los varones. Sin embargo, ya desde la niñez temprana las niñas tienen mayor cantidad de masa grasa relativa que los varones en todas las edades, constituyendo el porcentaje más alto, en relación con el peso corporal, lo que muestra la diferencia sexual en la composición corporal. Se estima que un niño al nacer tiene alrededor de 5 billones de adipocitos, que aumentan en la vida posnatal hasta alrededor de 30 a 50 billones en los individuos no obesos. Estas células representan un peso de $500 \mathrm{~g}$ con respecto al peso corporal total, tanto en varones como en mujeres y aumenta a aproximadamente $10 \mathrm{~kg}$ en los varones y $14 \mathrm{~kg}$ en las niñas al comienzo de la edad adulta. En cuanto al número total de células, éstas aumentan de tamaño (hipertrofia) de acuerdo con la ingesta calórica. Al llenarse y continuar con la ingesta elevada se estimula la división celular (mitosis) y, por ende, aumenta el número de adipocitos (hiperplasia) durante toda la vida. Durante el crecimiento la distribución corporal de la grasa también cambia. En todas las edades las niñas tienen mayor promedio de grasa subcutánea que los varones, especialmente en la adolescencia. La diferencia sexual es más evidente en la grasa subcutánea de las extremidades que en el tronco. Proporcionalmente los varones tienen más grasa subcutánea en el tronco en comparación con las extremidades que las niñas, cuya acumulación es precisamente en la zona gluteofemoral. Esta acumulación selectiva de adiposidad está controlada, en parte, por las hormonas sexuales. El metabolismo del tejido adiposo es complejo, y también intervienen en él la hormona de crecimiento, los glucocorticoides, la insulina y la hormona tiroidea. Además, es la primera fuente extragonadal de estrógenos.

Sistema nervioso. El desarrollo del equilibrio, agilidad y coordinación es indispensable para la adquisición y realización de los movimientos hábiles y reacciones rápidas. Para que esto ocurra, es necesario que la mielinización de las fibras nerviosas se haya completado, y esto sucede paulatinamente durante el período de crecimiento y desarrollo, observándose cómo la mielinización acelera la transmisión de los impulsos nerviosos. La mielinización de la corteza cerebral se desarrolla con mucha rapidez durante la infancia, pero continúa hasta mucho después de completada la pubertad. El desarrollo motor en la lactancia y la niñez temprana refleja la continua maduración neuromuscular, lo que está relacionado con el rápido crecimiento del cerebro anterior. El cerebelo, cuya función incluye el desarrollo y mantenimiento de la coordinación neuromuscular, el equilibrio y el tono muscular, tiene su brote de crecimiento más tarde de lo que lo hace el cerebro anterior y el tronco encefálico, pero completa su brote más tempranamente, ya que hacia los 18 meses de edad el contenido de células del cerebelo ya ha alcanzado el nivel adulto, mientras que el cerebro y el tronco encefálico sólo tienen un $60 \%$ del total del adulto. Es también durante este tiempo que el niño desarrolla el control postural y el equilibrio necesarios para la marcha independiente. En este momento ocurren también cambios en el número de células, la mielinización y la actividad cerebral. Cada área cerebral tendría su propio tiempo de desarrollo y habría al menos 3 períodos o más de maduración cerebral posnatal. El primero ocurre entre los 15 y 24 meses de edad, cuando casi todas las áreas presentan un estado de maduración similar. El segundo ocurre entre los 6 y 8 años, cuando la corteza cerebral sufre una remodelación con cambios en los pliegues y la densidad neuronal y, aunque no está claramente establecido, durante la adolescencia también existirían dos períodos de cambio en la corteza cerebral.

\section{Actividad, crecimiento y maduración.}

Todas las funciones orgánicas tienen períodos de 
maduración, de optimización, de mantenimiento y luego de declinación. Según algunos autores, los cambios que acompañan al niño y al adolescente deportista en su crecimiento y desarrollo se relacionan con la habilidad motora, la fuerza, la función pulmonar, la función cardiovascular, la capacidad aeróbica, la economía de carrera, la capacidad anaeróbica y la tensión térmica.

La habilidad motora. El desarrollo motor es el proceso por el cual un niño adquiere los patrones de movimiento y habilidades. El patrón motor tiene que ver con el movimiento básico (patrones motores fundamentales), mientras que la habilidad se refiere a la eficiencia del movimiento. Los patrones básicos fundamentales son formas elementales del movimiento que comprenden a las actividades locomotrices (caminar, correr, saltar, galopar, rodar, botar, caer, trepar, subir, bajar, etc.), las actividades no locomotrices (balancearse, estirarse, inclinarse, girar, empujar, doblarse, traccionar, colgarse, equilibrarse, etc.), en las que sólo alguna parte específica del cuerpo es movida, y las manipulativas o proyectivas, en las cuales se mueven objetos (arrojar, lanzar, atrapar, patear, recepcionar, batear, driblear, etc.). Estas actividades están presentes en todas las disciplinas deportivas como patrones motores sobre los que se construyen las técnicas específicas. Generalmente la mayoría de las habilidades motoras fundamentales se desarrollan a los $6-7$ años de edad, aunque los patrones de madurez de algunas de estas habilidades no se desarrollan hasta más tarde. Así, hay niños de $6-7$ años de edad que no han desarrollado suficientemente la coordinación y el control para cumplir satisfactoriamente las habilidades motoras fundamentales. El desarrollo motor en la infancia y niñez está relacionado con la maduración neuromuscular, el rápido crecimiento del sistema nervioso y, consecuentemente, la mielinización. Una vez que los patrones básicos de movimiento están establecidos, el aprendizaje y la práctica son factores que influyen significativamente sobre la competencia motora, además de las características de crecimiento y maduración del niño. Posteriormente, la actividad motora requerirá el esfuerzo del vigor o fuerza, la potencia y la velocidad. La habilidad motora aumenta con la edad, tanto en varones como en mujeres, hasta los 18 años, aunque en estas últimas tiende a estabilizarse hacia la pubertad, lo que se debería a las diferencias hormonales entre ambos sexos. El período ideal para el aprendizaje motor es el comprendido entre los 8 y los 12 años de edad.

Fuerza. Se llama fueza propiamente dicha a la capacidad para ejercer acción muscular contra una resistencia. La fuerza aumenta con la edad y lo hace de manera diferente en ambos sexos, a la vez que los patrones de progreso no son uniformes para todos los esfuerzos. La fuerza estática aumenta linealmente con la edad hasta los 13 - 14 años en los varones, momento en que se presenta un brote adolescente. En las niñas, la fuerza también progresa linealmente con la edad hasta los 16 o 17 años, pero no hay evidencia de que en ellas ocurra un brote adolescente, alcanzando el máximo alrededor de los 20 años. En los varones la fuerza continúa aumentando durante la tercera década de la vida, alcanzando su máximo entre los 20 y 30 años de edad. El desarrollo de la fuerza, la potencia y la habilidad no será posible si no se ha alcanzado la madurez nerviosa. La mielinización de muchos nervios motores es incompleta hasta la madurez sexual, por lo que el control neural de la función muscular es limitado antes de llegar a esta fase. En estudios longitudinales llevados a cabo entre los 7 y 18 años se encontró que la fuerza muestra un incremento alrededor de los 12 años en los varones, coincidiendo con el inicio de la pubertad. No se registran datos similares en las niñas.

Función pulmonar. La función pulmonar cambia más en función de la estatura que con respecto a la edad. Es decir, el incremento de los volúmenes pulmonares en la niñez y la adolescencia tiende a ser proporcional a la talla. Todos los volúmenes aumentan hasta que se alcanza la madurez física. La ventilación pulmonar máxima, en valores absolutos, aumenta con la edad y en valores relativos es igual en adultos, jóvenes y niños. La ventilación pulmonar submáxima disminuye con la edad, lo que sugiere una menor reserva ventilatoria en las edades infantiles. La ventilación pulmonar y el VO2 aumentan linealmente hasta el punto de "ruptura" respiratorio donde la ventilación pulmonar se acelera marcadamente, determinando una elevación del equivalente respiratorio debido a la necesidad de aumento de la cantidad de litros movilizados por cada litro de oxígeno consumido, por lo que el niño presentaría una ventilación antieconómica, ya que debe movilizar más aire por litro de oxígeno consumido. En comparación con los adultos y adolescentes, los niños responden al ejercicio con una alta frecuencia respiratoria (FR) y una ventilación superficial. Si analizamos el desarrollo de la maduración pulmonar, comprenderemos mejor lo mencionado. Aproximadamente a partir del noveno o décimo mes de la vida fetal comienza el desarrollo alveolar, pero la mayoría de los alvéolos se desarrollan y maduran después del nacimiento. El tejido pulmonar crece considerablemente en la vida posnatal y maduran 
progresivamente las funciones respiratorias. Los pulmones humanos pesan alrededor de $60-70 \mathrm{~g}$ al nacer y aumentan su masa alrededor de 20 pliegues antes de alcanzar la maduración, en contraste con el corazón, el cual crece en forma proporcional al aumento de estatura. El número de alvéolos aumenta desde aproximadamente 20 millones al nacimiento hasta 300 millones alrededor de los 8 años de edad, alcanzando en ese momento la cantidad que corresponde a la edad adulta.

Función cardiovascular. Como todas las funciones orgánicas, la función cardiovascular experimenta cambios durante todo el proceso de crecimiento y desarrollo. El tamaño del corazón es directamente proporcional al tamaño corporal y, por lo tanto, los niños tienen corazones más pequeños que los adultos. A consecuencia de esto y de un menor volumen sanguíneo, el niño tiene menor volumen sistólico. La mayor frecuencia cardíaca máxima del niño sólo puede compensar parcialmente esta menor capacidad del volumen sistólico y, por lo tanto, el gasto cardíaco máximo es menor que el de un adulto con un mismo nivel de entrenamiento. El mayor flujo sanguíneo muscular en los niños representa una distribución más favorable de la sangre durante el ejercicio. Esto facilita el transporte de oxígeno al músculo activo $y$, junto con el aumento de la diferencia arteriovenosa de oxígeno, compensa el bajo gasto cardíaco, pero no hay que olvidar que la capacidad del niño para liberar oxígeno es menor que en el adulto. El comportamiento de la presión arterial muestra que es directamente proporcional al tamaño corporal, es decir, es menor en los niños que en los adultos y va aumentando paulatinamente hasta alcanzar los niveles adultos alrededor de los 20 años de edad. La respuesta cardiovascular y pulmonar al ejercicio a una potencia submáxima determinada cambia con la edad. Sin embargo, los cambios que aparecen con la edad están más relacionados con la masa corporal o estadio de la maduración biológica que con la edad cronológica.

Capacidad aeróbica. La capacidad aeróbica consiste en acomodar la necesidad de oxígeno de los músculos que se ejercitan mediante la adaptación cardiovascular como respuesta al aumento de la actividad. El incremento en la función pulmonar y cardiovascular acompaña al crecimiento. De manera similar lo hace la capacidad aeróbica (VO2 máx). EI VO2 máx, expresado en litros por minuto $(\mathrm{I} / \mathrm{min})$, alcanza su nivel más alto entre los 17 y los 21 años de edad en los varones y entre los 12 y 15 años en las mujeres, disminuyendo luego en forma sostenida. La potencia aeróbica máxima muestra un claro brote adolescente al final del pico de máximo crecimiento. La actividad desarrollada por el individuo está directamente relacionada con la magnitud de la potencia aeróbica, lo que se advierte sobre todo en los varones, ya que antes, durante y después del brote de crecimiento puberal los varones activos tienen una potencia aeróbica máxima relativa mayor que los que tienen un nivel promedio de actividad física. Si relacionamos al VO2 máx con el peso corporal, se comprueba que en los varones se estabiliza desde los 6 hasta los 25 años; en cambio en las niñas su declinación comienza aproximadamente a los 13 años de edad, lo que se atribuye, entre otros factores, al aumento de la grasa corporal que se produce en ellas durante la pubertad. Esta relación del VO2 máx con el peso corporal puede no proporcionar una estimación precisa de la capacidad aeróbica. Aunque los incrementos del VO2 máx que acompañan al entrenamiento de resistencia en los niños son relativamente pequeños en comparación con los adultos, los incrementos en el rendimiento de estos niños son relativamente grandes. El menor valor del VO2 máx del niño ( $/ /$ min) limita la capacidad de resistencia, a menos que el peso corporal constituya la principal resistencia al movimiento, como en las carreras de fondo, en las que los niños no deberían estar en desventaja porque sus valores de VO2 máx expresados en relación con el peso corporal son ya los del adulto o similares. Aquí juega un papel principal la economía de esfuerzo, pues el niño tendrá un consumo de oxígeno submáximo sustancialmente más elevado que el adulto al considerarlo en relación con el peso corporal. Asimismo, los cambios físicos que se producen en el momento de la pubertad, como el alargamiento de las piernas y el fortalecimiento muscular, hacen que mejoren las técnicas al correr. Por lo tanto, la economía al correr aumenta. Esto mejora el ritmo en las carreras de fondo, aunque los niños no estén entrenados y sus valores de VO2 máximo no aumenten.

Capacidad anaeróbica. La capacidad anaeróbica es limitada en los niños. Ellos no pueden alcanzar concentraciones de ácido láctico en los músculos o en la sangre como los adultos, debido a una menor capacidad glucolítica, probablemente por una menor concentración de fosfofructocinasa, una enzima clave que controla el ritmo de la glucólisis. La potencia anaeróbica aumenta con el crecimiento y el desarrollo, y el entrenamiento anaeróbico mejoraría tal capacidad en los niños, mejorando los niveles en reposo de fosfocreatina, ATP y glucógeno, con mayor actividad de la fosfofructocinasa y, por lo tanto, con mayor nivel de lactato en sangre. La potencia 
anaeróbica generada por un niño de 8 años es el $70 \%$ de la que puede generar uno de 11 años.

Tensión térmica. De acuerdo con estudios llevados a cabo en laboratorio, se comprobó que los niños son más susceptibles de padecer enfermedades o lesiones inducidas por el frío o el calor que los adultos. Esto se debería a una menor capacidad del niño para perder calor por la sudoración cuando se ejercita en ambientes cálidos, porque sus glándulas sudoríparas forman sudor más lentamente. Además, su ritmo de aclimatación a los ambientes cálidos es menor que en los adultos. Los niños producen mayor calor metabólico por kilogramo de peso que los adolescentes o los adultos, lo que implica mayor esfuerzo de sus mecanismos termorreguladores. Los niños también están expuestos a sufrir hipotermia en ambientes fríos, es decir, no tienen la capacidad de regular la temperatura como el adulto. En las niñas la temperatura corporal puede ser mayor a la de los varones durante el ejercicio (en 2 ó $3^{\circ} \mathrm{C}$ ) debido a que poseen menor número de glándulas sudoríparas. Por lo tanto, con la actividad física producen y mantienen mayores temperaturas corporales, lo que puede causarles inconvenientes en días calurosos y húmedos.

Maduración biológica. Todo el período de crecimiento y desarrollo ocupa en el hombre más de la cuarta parte de su vida media. El $20 \%$ final de este crecimiento lo completa durante la adolescencia. Una característica destacada de este período de crecimiento y desarrollo es la gran variabilidad en la edad de iniciación de los cambios corporales y la aceleración de la velocidad de crecimiento. Las niñas maduran antes que los varones, se adelantan 2 años en el crecimiento óseo lineal, es decir, hacen su pico de máximo crecimiento 2 años antes que los varones, a una edad promedio de 12 años (de 14 años en los varones). La diferencia no es la misma para la adquisición de los caracteres sexuales secundarios, evaluados éstos de acuerdo con los criterios de Tanner. Con respecto a éstos y siempre refiriéndonos al promedio, los varones comienzan su desarrollo genital 8 meses después que las niñas iniciaron su desarrollo mamario. El vello pubiano aparece en ellos 1 año después que en las niñas. En las niñas la menarquia acontece justo en medio de la sucesión de hechos, a una edad promedio de 12.36 años. No obstante, la primera menstruación puede acontecer entre los 10,5 años y los 15,5 años. Si se calcula la composición corporal en el momento de la menarquia (según Frisch), ésta se presenta con una talla promedio de $156 \mathrm{~cm}$, $45,9 \mathrm{~kg}$, un porcentaje de grasa mínimo del $17 \%$ y un promedio del $23,2 \%$.
Erupción dentaria. La edad dental puede determinarse por el grado de formación o por la emergencia de los dientes. Junto con la edad ósea, la morfométrica y la sexual constituyen un medio eficaz de evaluación de la edad biológica (fisiológica) del niño. Al comparar ambos sexos se observa que en el maxilar superior el segundo molar permanente está presente en el $100 \%$ de los varones a los 16 años y en las niñas a los 16,5 años, mientras que en el maxilar inferior este elemento ya ha alcanzado ese porcentaje a los 14 años en las mujeres y a los 16 años en los varones. La erupción del tercer molar comienza a la misma edad en ambos sexos, pero las mujeres llegan a los 19 años con un porcentaje mayor de elementos presentes.

\section{Aspectos psicosociales.}

El deporte es una de las actividades que exigen simultáneamente la participación del cuerpo, la inteligencia y la voluntad. Nos permite canalizar nuestras energías y angustias. Nos debe hacer recordar que nuestros congéneres son compañeros de juego y no competidores o enemigos a vencer. La actividad física es un medio formativo inmerso en el contexto educativo total y un integrador de la personalidad.

Aunque los términos actividad física y deporte se usan con frecuencia de manera indistinta, el deporte tiene una dimensión social diferente a la primera. Las primeras etapas de la participación deportiva son en realidad un juego, pero, como también representan obstáculos que vencer, permiten comprobar cómo pueden ser superados. Estos obstáculos reflejan, al parecer, tanto un juego como una prueba personal. De esta manera los niños pueden aprender a manejar logros y fracasos, a entender el valor de la práctica y la preparación para mejorar el rendimiento, a entender el concepto de justo e injusto, aceptar la autoridad adulta e interrelacionarse con distintos grupos sociales y económicos, así como la autodisciplina.

Pero, además de los efectos positivos, el deporte puede tener efectos negativos, especialmente sobre aquellos niños que se destacan o muestran algunas condiciones especiales que los proyectan como un posible futuro talento. La presión que en reiteradas ocasiones es ejercida por los padres, entrenadores, profesores de educación física, etc. y el temor ante el menor fracaso pueden ayudar a disminuir su autoestima, provocarles estrés, angustia, hasta llegar incluso a la depresión y a sufrir lesiones físicas cada vez más frecuentes. Estos síntomas no 
advertidos exponen a los niños a la conducta negativa de los adultos.

Muy importante es diferenciar entre "iniciación deportiva" y "especialización deportiva". La primera se refiere al proceso de enseñanza aprendizaje en la que el niño o adolescente aprende las destrezas básicas de un deporte, mediante el juego y de acuerdo con las posibilidades. Especialización deportiva implica desarrollo y entrenamiento de las capacidades técnico-tácticas y físicas, con el fin de lograr el mayor rendimiento posible. Esto último se consigue en competencias regulares, según categorías establecidas, cumpliendo reglamentos, etc. Para conseguir esto debe existir una maduración física, neurológica, cognitiva y psicológica, así como un contexto social apropiado. Aunque hay una tendencia a que los niños participen en los deportes a edades tan tempranas, por ejemplo 5 años para natación, $4-5$ años para gimnasia y 6 años para fútbol, y aunque es difícil generalizar acerca de cuándo un niño está listo para participar en un deporte organizado, se habrá notado que en el sentido de la confrontación social ésta no se desarrolla totalmente antes de los 6 años.

La maduración cognitiva para entender la naturaleza competitiva del deporte no se desarrolla antes de los 8 o 9 años. Además, comprender conceptos tales como la posición dentro de un equipo, la posición y función dentro del campo de juego o cómo seguir las instrucciones y estrategias pueden aparecer aún más tarde. De modo que los niños pueden no alcanzar la madurez psicológica total para competir en los deportes hasta los 11 o 12 años.

Hay niños que sobresalen naturalmente en un deporte, por lo que los padres desean saber si podrán llegar a integrar el grupo de los deportistas de élite. Es muy difícil predecir lo que ocurrirá con ellos, porque se presentan numerosas variables que dificultan el pronóstico, ya que ellas son de naturaleza biológica, psicológica y sociocultural, y se relacionan con el rendimiento. También habrá que tener en cuenta las demandas específicas para cada deporte.

Asimismo, la experiencia en los deportes es percibida de manera distinta en las diferentes etapas adolescentes. Esto se relaciona con los diferentes grados de madurez psicológica y cognitiva en la adolescencia temprana, media y tardía. En la adolescencia temprana los jóvenes entienden conductas y consecuencias sobre una base de "aquí y ahora". En la adolescencia media ya desarrollan una perspectiva de tiempo futuro y de razonamiento abstracto, siendo importante para ellos la opinión de padres y entrenadores. En la adolescencia tardía perciben sus habilidades deportivas de manera más acorde con la realidad, al mismo tiempo que aparecen otros intereses que pueden ser más importantes para ellos que los deportes.

Los niños y adolescentes pueden sufrir lesiones por sobrecarga o estrés y responden a ellas según sus propias características. Aunque reaccionan mejor frente a una lesión, porque no dudan con respecto a su curación, a veces se presentan situaciones que los vuelven negativos, sobre todo si la lesión tarda en resolverse, lo que también depende del apoyo familiar recibido y si existe un estado de ansiedad o depresión subyacente.

Si bien la práctica de los deportes puede resguardar a niños y adolescentes de caer en conductas negativas, muchas veces, por distintos motivos, ellos participan en actos de agresión y violencia, más común en deportes de contacto, en el uso de sustancias prohibidas para mejorar su rendimiento o también en el uso de suplementos nutricionales para aumentar su masa muscular y fuerza, entre otros objetivos. El motivo por el cual un adolescente hace uso de estas sustancias se relacionan con la presión ejercida por su entorno (amigos, padres, entrenadores, etc.) para la consecución de determinados logros, el miedo a defraudar a los demás o a sí mismo y, también, con el estrés competitivo. Otra de las conductas negativas es la relacionada con el control del peso corporal, parámetro muy importante en la práctica de algunos deportes como los estéticos (ballet, patinaje artístico, gimnasia, etc.), y que puede llevar, sobre todo a las niñas, al padecimiento de algún desorden alimentario.

La participación intensiva en los deportes implica que el niño, en muchas ocasiones, inicie el entrenamiento específico a edades tan tempranas como los 5 o 6 años, y a veces antes. Este entrenamiento se lleva a cabo, a veces, en horarios extras, aparte de todas las otras actividades normales o sociales que debe realizar y, en muchas ocasiones, pueden conducir al padecimiento de problemas como cansancio y fatiga. Esto, a su vez, provoca lesiones por sobreuso en el sistema musculoesquelético. Toda esta sucesión de eventos puede producir un estado de ansiedad, depresión y desorden alimentario, además de conducir al niño o adolescente al síndrome de sobreentrenamiento y, como consecuencia final, al abandono del deporte. 
En cuanto a los efectos que la actividad física intensa puede provocar, particularmente en las niñas, lo más destacado es, respecto de la maduración biológica, un retraso en el desarrollo puberal y más específicamente retraso en la aparición de su primera menstruación o menarquia. Según Frisch, en un estudio realizado en adolescentes deportistas, la menarquia se retrasa 5 meses por cada año de entrenamiento antes de ella. Esto se relaciona con el menor peso corporal y el menor porcentaje de grasa corporal. Se requiere, como mínimo, $17 \%$ de grasa para que las niñas puedan iniciar sus ciclos menstruales. Los efectos del entrenamiento sobre la maduración biológica de los varones, en cambio, no se consideran habitualmente, quizás porque ellos son siempre más activos que las niñas y quizás porque no hay un parámetro tan fácil de objetivar como la menstruación.

Aún está muy arraigado en la actual sociedad deportiva agrupar a los niños por edad cronológica, actitud muy frecuente en la práctica de deportes populares, como fútbol, básquet, voleibol, etc., sin considerar los cambios biofísicos que se producen durante la adolescencia, con la variabilidad que la caracteriza. De esta manera es posible enfrentar a niños de la misma edad, por ejemplo de 12 o 13 años, en donde uno tiene aún todas las características infantiles y el otro está avanzado en su maduración, observándose una marcada diferencia de desarrollo entre estos grupos de jóvenes. Es decir, que las categorías deberían ser establecidas por tamaño corporal (maduración biológica) y no por edad cronológica. Al tener esto en cuenta se estarían previniendo daños físicos, psicológicos y el abandono del deporte por parte de los niños y adolescentes.

\section{BIBLIOGRAFÍA}

1. Díaz F, Mercado C, Troncoso I, Heusser F, Clavería C. Rol de la evaluación preparticipativa en adolescentes, en el diagnostico de enfermedades cardiovasculares y prevención de muerte súbita. Rev Med Chil. 2010; 138(2):223-32.

2. Aguilar A, Cosin J. Corazón y deporte. En: Rodes J, Guardia J. editores. Medicina Interna. $1^{a}$ edición. Barcelona-España: MASSON; 1998. p. 1048-51.

3. Asociación de medicina del deporte de Colombia. Manifiesto de actividad física para Colombia. $2^{\mathrm{a}}$ edición. Colombia: AMEDCO; 2002.

4. Azpitarte J. Pruebas de esfuerzo. En: Rodes J, Guardia J. editores. Medicina Interna. $1^{a}$ edición. Barcelona - España: MASSON; 1998. p. 846-9.

5. Zawadzki N. La practica deportiva en niños, niñas y adolescentes. Pediatria. 2004; 31(1):701-20.

6. Casasnovas $\mathrm{O}$, Yulitta $\mathrm{H}$, Turganti A. Certificación para la actividad física escolar y pre-participativa deportiva. Arch Argent Pediatr. 2001; 99(6):538-42.

7. Celaya S. Desnutrición. En: Rodes J, Guardia J. editores. Medicina Interna. $1^{\text {a }}$ edición. Barcelona-España: MASSON; 1998. p. 2819-30.

8. Chacón M, Ballesteros R, González M. Visita deportiva de no contraindicación. Revista Pediatría de Atención Primaria. $2001 ; 3(12): 639-60$.

9. Kroeger A, Luna R. La desnutrición. En: Kroeger A, Luna R, editores. Atención primaria de salud: principios y métodos. $1^{\mathrm{a}}$ edición. México: Panamericana de Salud y Editorial Pax México; 1987. p. 241-81.

10. Kroeger A, Luna R. Salud oral. En: Kroeger A, Luna R, editores. Atención primaria de salud: principios y métodos. $1^{a}$ edición. México: Panamericana de Salud y Editorial Pax México; 1987. p. 241-81.

11. Kroeger A, Luna R. Salud mental. En: Kroeger A, Luna R, editores. Atención primaria de salud: principios y métodos. $1^{\mathrm{a}}$ edición. México: Panamericana de Salud y Editorial Pax México; 1987. p. 241-281.

12. Instituto Nacional de Estadística (INE), Ministerio de Salud y Desarrollo. Encuesta Nacional de Demografía y Salud 2003. La Paz, Bolivia; 2003.

13. Leveroni AF, Abella I, Coronel AR. La aptitud física: un tema pediátrico. Rev Hosp Niños B Aires. 1995; 35(152):22-5.

14. Ministerio de Salud de la ciudad de México. Las actividades competitivas. $2^{\mathrm{a}}$ edición. México: Editorial Interamericana; 2004.

15. Sacristán A, Temboury MC, Fernández A. Tratamiento de la obesidad infantil en atención primaria. Pediatrika. 2001; 3(5):65-71.

16. Surós J, Surós A. Semiología médica y técnica exploratoria. $7^{\text {a }}$ edición. México, D.F.: Salvat Editores; 1987.

17. Totoro A. Aspectos pediátricos del examen físico preparticipativo. Rev Arg Med Deporte AMMDEP. 1999; 66(21):35-6.

18. Egocheaga J. Salud y deporte en la adolescencia. Bol Pediatr. 2007; 47(1):5-7. 